\title{
Chest CT in COVID-19 pneumonia: what are the findings in mid-term follow-up?
}

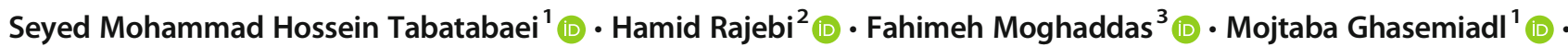 \\ Hamidreza Talari ${ }^{1}$ (D)
}

Received: 17 September 2020 / Accepted: 1 November 2020 / Published online: 9 November 2020

(C) American Society of Emergency Radiology 2020

\begin{abstract}
Purpose The purposes of this study are to investigate mid-term chest computed tomography (CT) findings of coronavirus disease 2019 (COVID-19) pneumonia, assess the rate of complete resolution, and determine the individuals at risk for residual abnormalities.

Methods Fifty-two cases of COVID-19 pneumonia with at least two chest CTs and mean 3-month interval between the initial and follow-up CT were enrolled in this retrospective study. Patients were categorized into two groups: complete resolution and residual disease on follow-up CT. Demographic, clinical, laboratory, and therapeutic data as well as initial and follow-up chest CT scans were compared and analyzed.

Results Thirty patients $(57.7 \%)$ demonstrate complete resolution of pulmonary findings, and 22 patients $(42.3 \%)$ had residual disease on follow-up CT. The mean time interval between initial and follow-up CT was $91.3 \pm 17.2$ and $90.6 \pm 14.3$ days in the complete resolution and residual groups, respectively. The most common radiologic pattern in residual disease was ground-glass opacities $(54.5 \%)$, followed by mixed ground-glass and subpleural parenchymal bands $(31.8 \%)$, and pure parenchymal bands $(13.7 \%)$. Compared to complete resolution group, patients with residual disease had higher CT severity score on initial exam $(10.3 \pm 5.4$ vs. $7.3 \pm 4.6, P$ value $=0.036)$, longer duration of hospitalization, higher rate of intensive care unit (ICU) admission, more underlying medical conditions, higher initial WBC count, and higher occurrence rate of leukocytosis in the hospitalization time period (all $P$ values $<0.05$ ).

Conclusion Extensive lung involvement on initial CT, ICU admission, long duration of hospitalization, presence of underlying medical conditions, high initial WBC count, and development of leukocytosis during the course of disease are associated with more prevalence of chronic lung sequela of COVID-19.
\end{abstract}

Keywords COVID-19 $\cdot$ Pneumonia $\cdot$ CT $\cdot$ Follow-up $\cdot$ Mid-term $\cdot$ Sequela

Seyed Mohammad Hossein Tabatabaei and Hamid Rajebi contributed equally to this work.

Seyed Mohammad Hossein Tabatabaei

hoseintabatabaii@gmail.com; tabatabaei-smh@kaums.ac.ir

1 Radiology Department, Faculty of Medicine, Kashan University of Medical Sciences, Kashan, Iran

2 Radiology Department, Massachusetts General Hospital, Harvard Medical School, Boston, MA, USA

3 Faculty of Medicine, Kashan University of Medical Sciences, Kashan, Iran

\section{Introduction}

Novel coronavirus disease 2019 (COVID-19) has become a global pandemic. As of October 26, 2020, more than fortytwo million confirmed cases have been reported worldwide, with more than 1.15 million deaths, indicating an approximate mortality rate of $2.7 \%$ [1].

Reverse transcription polymerase chain reaction (RT-PCR) and chest computed tomography (CT) scan are the main tools for diagnosis of severe acute respiratory syndrome coronavirus 2 (SARS-CoV-2) infection, the causal agent of COVID19 , with a reported sensitivity of $97 \%$ for CT scan $[2,3]$. While laboratory-based performance evaluations of RT-PCR tests show high analytical sensitivity and near-perfect specificity with no misidentification of other coronaviruses or 
common respiratory pathogens, test sensitivity in clinical practice may be adversely affected by a number of variables, including adequacy of specimen, specimen type, specimen handling, and stage of infection when the specimen is acquired (Centers of Disease Control guidelines for in vitro diagnostics) $[4,5]$.

According to a multinational consensus guideline from the Fleischner Society, imaging is indicated for patients with moderate-to-severe features or those with evidence of worsening respiratory status [6]. CT is indicated in patients with functional respiratory impairment and/or hypoxemia after recovery from COVID-19 [6]. CT scan has been used not only for the initial assessment of the lung involvement and its extent but also to predict the prognosis and mortality, identify the complications, guide treatment plans, and monitor the pulmonary findings of the patients after treatment [7-10]. In order to reduce the radiation dose and cancer risk, chest $\mathrm{CT}$ can be performed by low-dose protocols in COVID-19 patients with high accuracy [11].

Some recently published studies have focused on shortterm imaging follow-up of patients with COVID-19 pneumonia, describing the temporal progression of $\mathrm{CT}$ findings and subsequent resolution or persistence of them [9, 12-16]. For example, a study by Liu N. et al. on the relationship between dynamic changes of chest CT follow-up in COVID-19 and clinical typing of the disease showed that the occurrence rate of ground-glass opacity, crazy paving pattern, pleural thickening, or adhesion is higher in severe and critical group [17]. Although some of these reports suggest that chest CT scan findings of patients are improving with proper care, there are still studies that demonstrate many abnormal imaging findings at the time of discharge, including evidence of fibrosis [13, 18]. For example, a study by Huang et al. on follow-up COVID-19 patients 30 days after discharge showed that more than half of the patients still had residual abnormalities on CT scans, and in cases with initial severe pulmonary involvement, fibrosis was observed at the site of involvement [18].

A mid-term follow-up study of chest CT in COVID-19 pneumonia allows the identification and risk stratification of chronic radiologic findings in COVID-19 pneumonia, and potential recommendation for continued radiological surveillance. High-resolution chest CT in COVID-19 may be indicated not only for characterization of pulmonary abnormalities in patients with abnormal chest X-ray but also to establish a baseline for possible future follow-up [19].

In this retrospective study, we aim to investigate mid-term (an average of 3 months) follow-up CT scan findings of COVID-19 pneumonia, assess the rate of complete resolution of the initial CT findings, and determine the individuals who are at risk for possible residual abnormalities. We hypothesized that certain demographic, clinical, laboratory, and imaging characteristics may predict the likelihood of residual parenchymal findings on chest CT of COVID-19 patients.

\section{Methods}

\section{Patient selection}

This retrospective study was approved by the ethics committee of Kashan Universiy of Medical Sciences (approval ID: IR.KAUMS.REC.1399.007) and in accordance with the 1964 Helsinki Declaration and its later amendments or comparable ethical standards. Medical records of all patients with RT-PCR confirmed COVID-19 who underwent chest CT scan in Shahid Beheshti Hospistal, Kashan, Iran from February 18 to July 2, 2020, were assessed. Patients who had at least two chest CT scans with more than 75-day interval between the initial and the last CT were identified as eligible subjects to be enrolled in our study. For patients with more than two chest CTs, the initial CT was defined as the CT with the highest CT severity score [14] during hospitalization or acute phase of illness, and the follow-up CT was defined as the last CT recorded. Finally, 52 patients were enrolled in the study. The indications of performing the follow-up scan included referring by the clinician either for being symptomatic and functional impairment after recovery from COVID19 (just as Fleischner society recommendation for COVID-19 management) [6] or because of patient's concern about the COVID-19 sequela. The relevant demographic, clinical, laboratory, and therapeutic data were also collected.

\section{Demographic, clinical, laboratory, and therapeutic data}

Upon review of patients' medical record, demographic, clini$\mathrm{cal}$, and laboratory data, including dominant symptoms on initial presentation and at the time of follow-up, body mass index (BMI), initial $\mathrm{SpO}_{2}$, white blood cell (WBC) count, quantitative C-reactive protein (CRP), lactate dehydrogenase (LDH) level, and the presence or absence of leukocytosis in course of hospitalization (if admitted), were collected. Outpatient or inpatient management, history of admission to intensive care unit and orotracheal intubation, and duration of hospitalization were also taken. Major underlying medical conditions (including cardiovascular, pulmonary, renal, or immunosuppressive diseases; hypertension; diabetes mellitus; or malignancy) and history of tobacco were also recorded. Also, therapeutic regimens in addition to supportive therapy were documented if administered. Of note, patients received standard institutional care for COVID-19 pneumonia, based on BMJ best practice and Massachusetts General Hospital guidelines, which included prophylactic anticoagulant for the admitted patients [20, 21]. 


\section{CT protocol}

All CT scans were performed using a multidetector scanner (Alexion TSX-034A, Canon, Japan). The scans were acquired and reconstructed as axial images using the following parameters: tube voltage: $120 \mathrm{kVp}$; tube current: $50-90 \mathrm{mAs}$ with automatic exposure control, slice thickness: $1 \mathrm{~mm}$, pitch factor: 1. CT images were acquired at full inspiration with the patient installed in the supine position, and without administration of intravenous contrast medium. The mean CTDIvol was 4.6 mGy (range: 3.3-6.8 mGy).

\section{CT interpretation}

There were 104 series of images from 52 eligible patients that were extracted and coded randomly by a radiologist who was not involved in final image interpretation. The patients' name was masked to make the readers blind. The images were read independently by two radiologists (reader A: SMH. T with 8year experience, and reader B: H.R. with 6-year experience) with a consensus approach. All CT images were viewed with both lung (width, $1600 \mathrm{HU}$; level, $-550 \mathrm{HU}$ ) and mediastinal (width, $400 \mathrm{HU}$; level, $40 \mathrm{HU}$ ) window settings.

A semiquantitative scoring system was used to estimate the extension of pulmonary involvement of all the mentioned abnormalities based on the area involved [14]. Each of the five pulmonary lobes was visually scored from 0 to 5 as $(0)$ no involvement, (1) less than 5\% involvement, (2) 5-25\% involvement, (3) $26-50 \%$ involvement, (4) $51-75 \%$ involvement, and (5) 76-100\% involvement. The scores were added together to provide a total CT severity score ranging from 0 (no involvement) to 25 (maximum involvement). In a qualitative evaluation, CT severity scores of $1-5,6-14$, and $15-25$ were categorized as mild, moderate, and severe involvement, respectively.

For each chest $\mathrm{CT}$ scan, the type of dominant finding was recorded. The major CT findings were described based on Fleischner Society glossary of terms for thoracic imaging [22]. The readers reported dominant pulmonary lesions as ground-glass opacity (GGO) with or without visible intralobular lines, consolidation, mixed consolidation and GGO (including reverse halo sign), and linear opacities (including perilobular pattern) [23].

Ultimately, based on the follow-up CT interpretation, we categorized the patients into two groups: patients who demonstrated complete resolution of the pulmonary findings on their follow-up CT $(\mathrm{CT}$ severity score $=0)$, and patients who had residual disease on their follow-up CT.

\section{Statistical analysis}

Categorical variables were presented as numbers and percentages. Continuous variables were presented as mean \pm standard deviation. Differences between two groups were analyzed by chi-square for categorical variables and one-way ANOVA for the continuous variables. All statistical analyses were performed with SPSS software (version 24.0, IBM Corp., Armonk, NY, USA) and $P$ values $<0.05$ were considered statistically significant.

\section{Results}

Of the 52 patients, 32 were male and 20 were female. The patients' ages ranged from 21 to 78 years with a mean of $50.17 \pm 13.1$. All patients were symptomatic on the initial presentation with laboratory confirmation of COVID-19. All demographic, clinical, laboratory, and initial/follow-up CT features and therapeutic regimens in our cohort of patients have been listed in Tables 1, 2, and 3, including the data on the independent reading by the two radiologists before consensus. The most common symptoms on initial presentation were fever $(46 / 52,88.4 \%)$, fatigue $(28 / 52,53.8 \%)$, and dyspnea (21/ $52,40.4 \%)$. The most common radiological pattern on initial CT was GGO (24/52, 46.2\%), followed by consolidation (14/ $52,26.9 \%)$ and mixed pattern $(14 / 52,26.9 \%)$.

The mean time interval between the initial and follow-up CT was $91 \pm 15.5$ days (ranging from 75 to 114 days). On follow-up CT, 30 patients (57.7\%) demonstrated complete resolution of pulmonary parenchymal lesions 30 (Fig. 1), and 22 patients $(42.3 \%)$ demonstrated residual disease (Figs. 2 and 3). The mean time interval between the initial and follow-up CT in complete resolution and residual disease groups was $91.3 \pm 17.2$ (range: $75-114$ ) and $90.6 \pm 14.3$ (range: $79-110)$ days, respectively $(P$ value $=0.852)$.

Ten $(33.3 \%)$ of the 30 patients with complete resolutions were symptomatic at the time of follow-up chest CT, including four (13.3\%) patients with mild chest pain, and six (20\%) with a vague feeling of discomfort in the chest. Of the patients with residual disease on follow-up CT, 54.5\% (12/22) were symptomatic, including six patients $(6 / 22,27.3 \%)$ with dyspnea on exertion, five patients $(5 / 22,22.7 \%)$ reporting mild chest pain or discomfort, and one patient $(1 / 22,4.5 \%)$ with occasional cough. The difference in being symptomatic between the resolved and residual group was not statistically significant $(P$ value $=0.125)$.

Patients who demonstrated residual disease on follow-up had a statistically significant higher initial CT severity score when compared to the group who had resolved disease (10.3 \pm 5.4 vs. $7.3 \pm 4.6, P$ value $=0.036$ ). In a qualitative assessment, the residual group on follow-up CT had a higher number of cases with severe involvement of the lungs when compared to the resolved group $(8 / 22,36.4 \%$ vs. $2 / 30,6.7 \%, P$ value $=0.011$.

The most common radiologic pattern in the patients with residual disease were GGO $(12 / 22,54.5 \%)$, followed by 
Table 1 Demographic and clinical characteristics of the patients

\begin{tabular}{|c|c|c|c|}
\hline & $\begin{array}{l}\text { Complete resolution of pulmonary } \\
\text { findings on f/u chest CT }(n=30)\end{array}$ & $\begin{array}{l}\text { Residual pulmonary findings on } \\
\text { f/u chest CT }(n=22)\end{array}$ & $P$ value \\
\hline Age & $50.5 \pm 13.0$ & $49.72+13.1$ & 0.831 \\
\hline \multirow[t]{2}{*}{ Gender } & Female: $14(46.7 \%)$ & Female: $6(27.3 \%)$ & 0.152 \\
\hline & Male: $16(53.3 \%)$ & Male: $16(72.7 \%)$ & \\
\hline \multirow[t]{2}{*}{ Inpatient vs. outpatient } & Inpatient: $23(76.7 \%)$ & Inpatient: $19(86.4 \%)$ & 0.384 \\
\hline & Outpatient: $7(23.3 \%)$ & Outpatient: $3(13.6 \%)$ & \\
\hline ICU hospitalization & $2(6.7 \%)$ & $9(40.9 \%)$ & 0.002 \\
\hline Duration of hospitalization, days (mean $\pm \mathrm{SD}$ ) & $6.9 \pm 3.4$ & $9.3 \pm 4.7$ & 0.031 \\
\hline At least one underlying medical condition & $5(16.7 \%)$ & $12(54.4 \%)$ & 0.004 \\
\hline Heart disease & 2 & 4 & 0.382 \\
\hline Diabetes mellitus & 1 & 3 & 0.299 \\
\hline Hypertension & 0 & 2 & N/A \\
\hline Pulmonary diseases & 1 & 1 & 1 \\
\hline More than one of above & 1 & 2 & 0.567 \\
\hline Tobacco smoker & $4(13.3 \%)$ & $4(18.2 \%)$ & 0.631 \\
\hline Symptoms on follow-up & $10(33.3 \%)$ & $12(54.5 \%)$ & 0.125 \\
\hline BMI & $24.6 \pm 3.4$ & $25.5 \pm 4$ & 0.386 \\
\hline Obese $(\mathrm{BMI} \geq 30)$ & $5(16.7 \%)$ & $6(27.2 \%)$ & 0.355 \\
\hline Overweight or obese $(\mathrm{BMI} \geq 25)$ & $14(46.7 \%)$ & $12(54.5 \%)$ & 0.573 \\
\hline
\end{tabular}

$I C U$ intensive care unit, $B M I$ body mass index

Table 2 Initial and follow-up chest CT features of the patients

\begin{tabular}{|c|c|c|c|}
\hline & $\begin{array}{l}\text { Complete resolution of pulmonary } \\
\text { findings on } \mathrm{f} / \mathrm{u} \text { chest } \mathrm{CT}(n=30)\end{array}$ & $\begin{array}{l}\text { Residual pulmonary findings } \\
\text { on } \mathrm{f} / \mathrm{u} \text { chest } \mathrm{CT}(n=22)\end{array}$ & $P$ value \\
\hline Initial CT severity score (mean $\pm \mathrm{SD})$ & $\begin{array}{l}7.3 \pm 4.6 \\
\left(\mathrm{~A}^{*}: 7.2 \pm 4.5, \mathrm{~B}^{*}: 7.6 \pm 4\right)\end{array}$ & $\begin{array}{l}10.3 \pm 5.4 \\
(\mathrm{~A}: 10 \pm 5.3, \mathrm{~B}: 10.8 \pm 5.9)\end{array}$ & 0.036 \\
\hline \multirow[t]{3}{*}{ Initial CT qualitative assessment of extension } & $\begin{array}{l}\text { Mild: } 13(43.3 \%) \\
\text { (A: } 13[43.3 \%] \text {, B: } 13[43.3 \%] \text { ) }\end{array}$ & $\begin{array}{l}\text { Mild: } 6(27.2 \%) \\
\text { (A: } 6[27.2 \%], \text { B: } 6[27.2 \%])\end{array}$ & 0.235 \\
\hline & $\begin{array}{l}\text { Moderate: } 15(50 \%) \\
\text { (A: } 16[53.3 \%] \text {, B: } 15[50 \%])\end{array}$ & $\begin{array}{l}\text { Moderate: } 8(36.4 \%) \\
\text { (A: } 9[40.1 \%], \text { B: } 8[36.4 \%])\end{array}$ & 0.328 \\
\hline & $\begin{array}{l}\text { Severe: } 2(6.7 \%) \\
\text { (A: } 1[3.3 \%], \text { B: } 2[6.7 \%])\end{array}$ & $\begin{array}{l}\text { Severe: } 8(36.4 \%) \\
\text { (A: } 7[31.8 \%], \text { B: } 8[36.4 \%])\end{array}$ & 0.011 \\
\hline \multirow[t]{3}{*}{ Dominant initial CT finding } & $\begin{array}{l}\text { GGO: } 13(43.3 \%) \\
\text { (A: } 13[43.3 \%], \text { B: } 11[36.7 \%] \text { ) }\end{array}$ & $\begin{array}{l}\text { GGO: } 11(50 \%) \\
\text { (A: } 11[50 \%], \text { B: } 10[45.4 \%])\end{array}$ & 0.634 \\
\hline & $\begin{array}{l}\text { Consolidation: } 9(30 \%) \\
\text { (A: } 9[30 \%], \text { B: } 10[33.3 \%])\end{array}$ & $\begin{array}{l}\text { Consolidation: } 5(22.7 \%) \\
\text { (A: } 5 \text { [22.7\%], B: } 6[27.3 \%] \text { ) }\end{array}$ & 0.559 \\
\hline & $\begin{array}{l}\text { Mixed pattern: } 8(26.7 \%) \\
\text { (A: } 8[26.7 \%], \text { B: } 9[30 \%])\end{array}$ & $\begin{array}{l}\text { Mixed pattern: } 6(27.3 \%) \\
\text { (A: } 6[27.3 \%], \text { B: } 6[27.3 \%])\end{array}$ & 0.961 \\
\hline Bilateral involvement on initial $\mathrm{CT}$ & $\begin{array}{l}22(73.3 \%) \\
\text { (A: } 21[70 \%], \text { B: } 23[76.7 \%])\end{array}$ & $\begin{array}{l}18(81.2 \%) \\
\text { (A: } 18[81.2 \%], \text { B: } 18[81.2 \%] \text { ) }\end{array}$ & \\
\hline Number of involved lobes on initial CT (mean $\pm \mathrm{SD})$ & $\begin{array}{l}3.2 \pm 1.2 \\
(\mathrm{~A}: 3.1 \pm 1.2, \mathrm{~B}: 3.3 \pm 1.3)\end{array}$ & $\begin{array}{l}3.5 \pm 1.3 \\
(\mathrm{~A}: 3.5 \pm 1.3, \text { B } 3.6 \pm 1.3)\end{array}$ & \\
\hline Follow-up CT severity score (mean $\pm \mathrm{SD})$ & $\mathrm{N} / \mathrm{A}$ & $\begin{array}{l}3.9 \pm 2 \\
(A: 3.5 \pm 2.1, B: 4 \pm 2)\end{array}$ & N/A \\
\hline Dominant follow-up CT finding & N/A & $\begin{array}{l}\text { GGO: } 12(54.5 \%) \\
\text { (A: } 12[50 \%], \text { B: } 12[\%]) \\
\text { GGO + linear: } 7(31.8 \%) \\
\text { (A: } 7[31.8 \%], \text { B: } 7[31.8 \%]) \\
\text { Linear: } 3(13.7 \%) \\
\text { (A: } 3[13.7 \%], \text { B: } 3[13.7 \%])\end{array}$ & $\mathrm{N} / \mathrm{A}$ \\
\hline Time interval between CTs, days (mean $\pm \mathrm{SD}$ ) & $91.3 \pm 16.9$ & $90.5 \pm 14$ & 0.852 \\
\hline
\end{tabular}

$G G O$ ground-glass opacity

*A and $\mathrm{B}$ refer to radiologist $\mathrm{A}$ and radiologist $\mathrm{B}$ 
Table 3 laboratory and therapeutic information of the patients

\begin{tabular}{llll}
\hline & $\begin{array}{l}\text { Complete resolution of pulmonary } \\
\text { findings on f/u chest CT }(n=30)\end{array}$ & $\begin{array}{l}\text { Residual pulmonary findings } \\
\text { on f/u chest CT }(n=22)\end{array}$ & $P$ value \\
\hline Initial $\mathrm{SpO}_{2}$, percentage (mean $\left.\pm \mathrm{SD}\right)$ & $91.3 \pm 2.9$ & $90.7 \pm 2.1$ & 0.414 \\
Initial WBC count (mean $\pm \mathrm{SD})$ & $5282.6 \pm 2784.5$ & $7211.7 \pm 2516.5$ & 0.017 \\
Development of leukocytosis & $2(6.7 \%)$ & $7(31.2 \%)$ & 0.018 \\
$\mathrm{CRP}, \mathrm{mg} / \mathrm{L}$ (mean $\pm \mathrm{SD})$ & $30.1 \pm 25.1$ & $37 \pm 23.8$ & 0.326 \\
$\mathrm{LDH}$, units/L $($ mean $\pm \mathrm{SD})$ & $701.6 \pm 231.1$ & $706.1 \pm 254.8$ & 0.947 \\
Medications other than supportive management & Hydroxychloroquine: $28(93.3 \%)$ & Hydroxychloroquine: $19(86.4 \%)$ & 0.3990 .8790 .9610 .495 \\
& Lopinavir/ritonavir: $13(43.3 \%)$ & Lopinavir/ritonavir: $10(45.5 \%)$ & Ribavirin: $6(27.3 \%)$ \\
& Ribavirin: $8(26.7 \%)$ & Corticosteroids*: $7(31.8 \%)$
\end{tabular}

$C R P$ C-reactive protein, $L D H$ lactate dehydrogenase

*Dexamethasone or methylprednisolone

mixed GGO and subpleural parenchymal bands $(7 / 22$, $31.8 \%)$, and pure subpleural parenchymal bands $(3 / 22$, $13.7 \%$ ) (Figs. 2 and 3). Of the total 19 patients with GGO on follow-up exam, in six cases, the location of GGO was only at the dependent portion of the lung(s), which potentially could be misinterpreted as dependent atelectasis. However, the local nature and patchy appearance of the observed lesions made it somewhat possible to differentiate from dependent atelectasis.

There was no statistically significant difference between resolved and residual groups in the rate of hospitalization $(23 / 30,76.7 \%$ vs. $19 / 22,86.4 \%$ respectively, $P$ value $=$ $0.384)$. These numbers were also true about receiving prophylactic anticoagulant, because it was given to all admitted patients but not to the outpatients. However, patients with residual disease on follow-up CT had a longer duration of hospitalization $(9.3 \pm 4.7$ days vs. $6.9 \pm 3.4$ days, $P$ value $=0.031)$ and a higher rate of ICU admission with endotracheal intubation on their initial admission $(9 / 22,40.9 \%$ vs. $2 / 30,6.7 \%, P$ value $=0.002$ )

Twelve patients $(12 / 22,54.4 \%)$ of the residual disease group had at least one underlying medical condition. That was statistically higher than the number of patients with underlying condition(s) in the complete resolution group $(5 / 30,16.7 \%)(P$ value $=0.004)$. There was no statistically significant difference in the number of tobacco smokers between the two groups $(4 / 30,13.3 \%$ in the complete resolution group vs. $4 / 22,18.2 \%$ in the residual disease group, $P$ value $=$ 0.631). Also, mean $\mathrm{SpO}_{2}$ on initial evaluation was not statistically different between the complete resolution and residual disease group $(91.3 \pm 2.9$ vs. $90.7 \pm 2.1$ respectively, $P$ value $=$ 0.414). Patients who later demonstrate residual disease on follow-up CT had higher initial WBC count $(7211.7 \pm$ 2516.5 vs. $5282.6 \pm 2784.5, P$ value $=0.017$ ) and higher rate of occurrence of leukocytosis during the course of hospitalization (if admitted) (7/22, 31.2\% vs. $2 / 30,6.7 \%$ ). Although $\mathrm{CRP}$ and $\mathrm{LDH}$ levels were also higher in residual disease group (CRP: $37 \pm 23.8$ vs. $30.1 \pm 25.1, P$ value $=0.326$; LDH: $706.1 \pm 254.8$ vs. $701.6 \pm 231.1, P$ value $=0.947)$, it did not reach a statistically significant difference.

In terms of therapeutic regimens, special drugs other than supportive medications were analyzed. The complete resolution and residual disease groups did not show statistically significant difference in receiving hydroxychloroquine (28/ $30,93.3 \%$ vs. $19 / 22,86.4 \%$, respectively, $P=0.399)$, lopinavir/ritonavir (Kaletra) (13/30, 43.3\% vs. 10/22, 45.5\%,
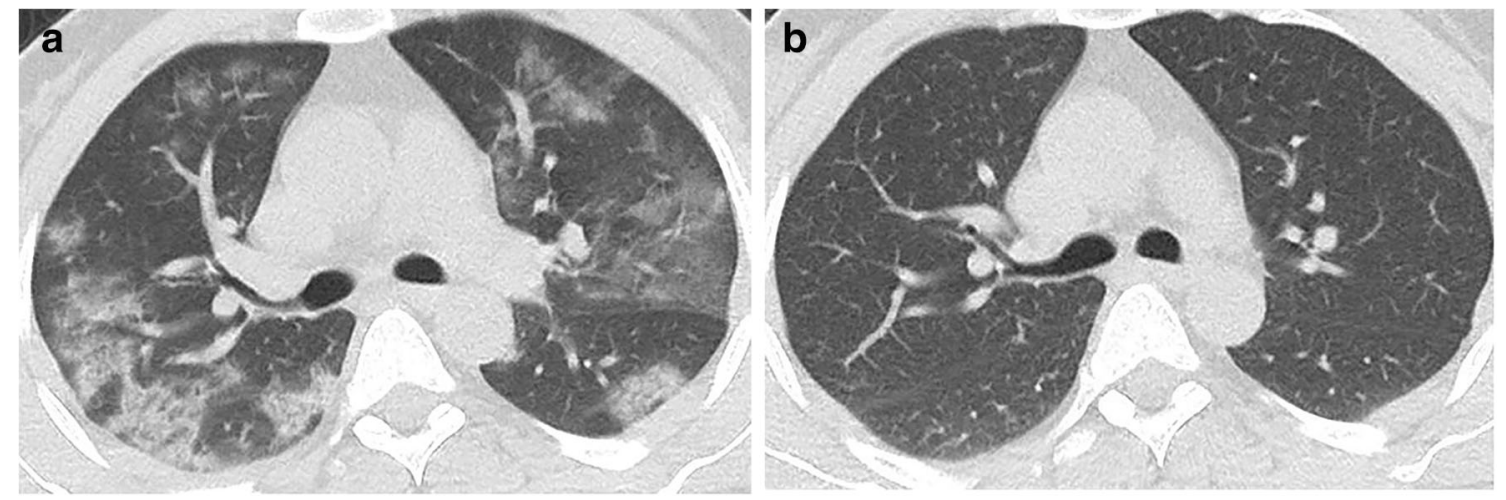

Fig. 1 Initial (a) and follow-up (b) chest CT scan after 83 days in a 45-year-old man with confirmed COVID-19 pneumonia with an initial CT severity score of 18 . Note the complete resolution of the pulmonary lesions on follow-up CT 

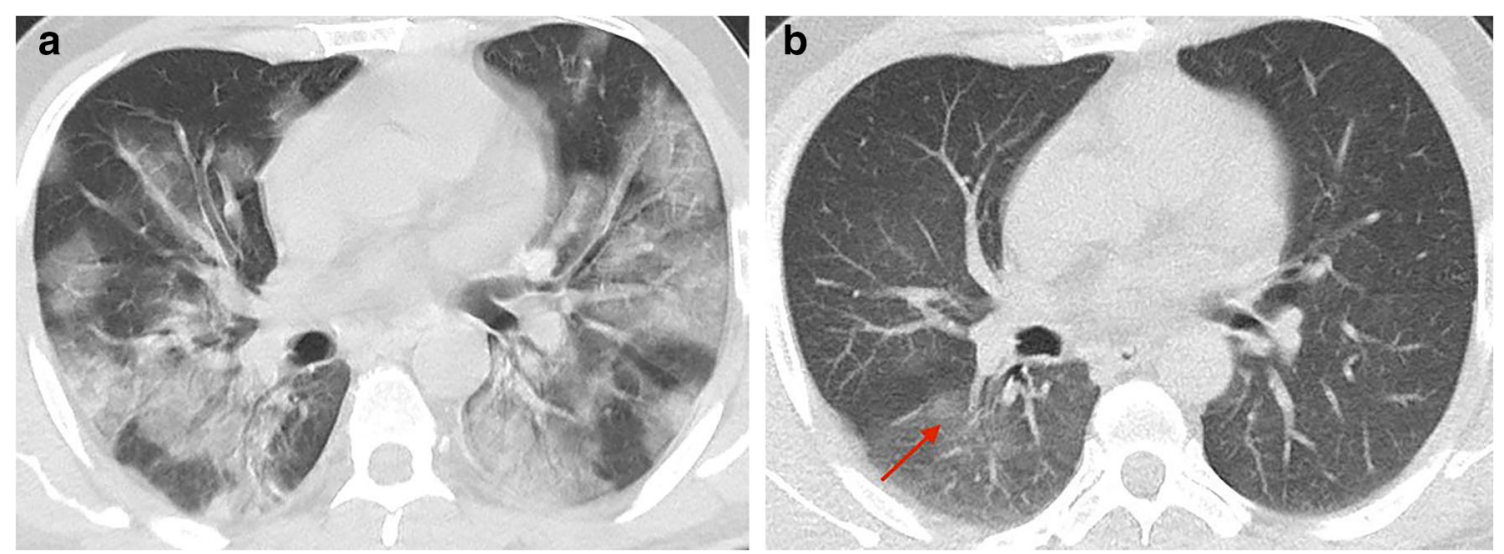

Fig. 2 Initial (a) and follow-up (b) chest CT scan after 102 days in a 55-year-old man with confirmed COVID-19 pneumonia with an initial CT severity score of 17. Note the residual disease as an area of ground-glass opacity in the superior segment of right lower lobe (arrow)

$P=0.879)$, ribavirin $(8 / 30,26.7 \%$ vs. $6 / 22,27.3 \%, P=$ $0.961)$, or corticosteroids $(7 / 30,23.3 \%$ vs. $7 / 22,31.8 \%, P=$ $0.495)$.

\section{Discussion}

SARS-CoV-2 infection and particularly its associated pneumonia poses a huge threat to global health. Many pulmonary and extrapulmonary manifestations of the infection have been described in the acute phase of the disease; however, data on mid-term imaging sequela are lacking. Although a few recently published studies have assessed the temporal course of COVID-19 pneumonia, almost all of them have investigated the progression of the disease in a short time interval, majority less than 30 days after the initial admission or presentation [9, 12-16].

Our retrospective analysis focuses on a mid-term follow-up (a mean of 3 months) in patients with confirmed COVID-19 pneumonia, the first radiology-related cohort in this time interval. A mid-term follow-up study opens a debate in the literature regarding who and at what stage a patient may need a

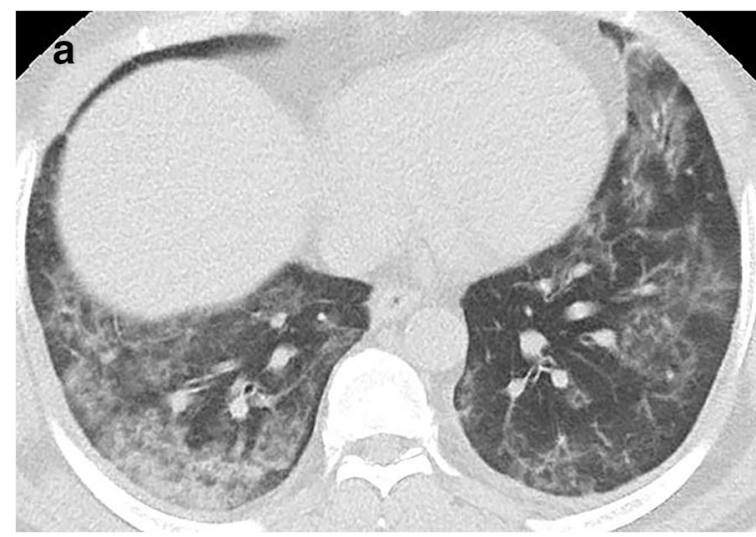

Fig. 3 Initial (a) and follow-up (b) chest CT scan after 108 days in a 45year-old man with confirmed COVID-19 pneumonia with initial CT severity score of 17 . Note the subpleural parenchymal bands (arrow) in the surveillance imaging in the era of ongoing COVID-19 pandemic. At the same time, chronic sequela of COVID-19 pneumonia raises the question of what kind of treatment should these patients be considered for if symptomatic. As there is a sharp rising curve of confirmed cases globally, the chronic health burden of the disease should be in the interest of health policymakers. Last but not the least, a follow-up study allows the risk stratification of the patients in the mid-term and identifies the patients at risk of developing the residual disease.

Our comparative analysis demonstrated that patients who had residual pulmonary parenchymal disease on a 3-month follow-up had a significantly higher rate of ICU admission, longer duration of hospitalization, more severe (extensive) disease on initial CT, more underlying medical conditions, higher WBC count on initial admission or presentation, and higher occurrence rate of leukocytosis during the time period of hospitalization; all of them are potential predictors of chronic lung sequala. Although there was no statistically significant difference in number of symptomatic patients between the two groups, dyspnea on exertion was reported only in the residual disease group and this symptom may be screened in the followup visits although more dedicated studies are warranted.

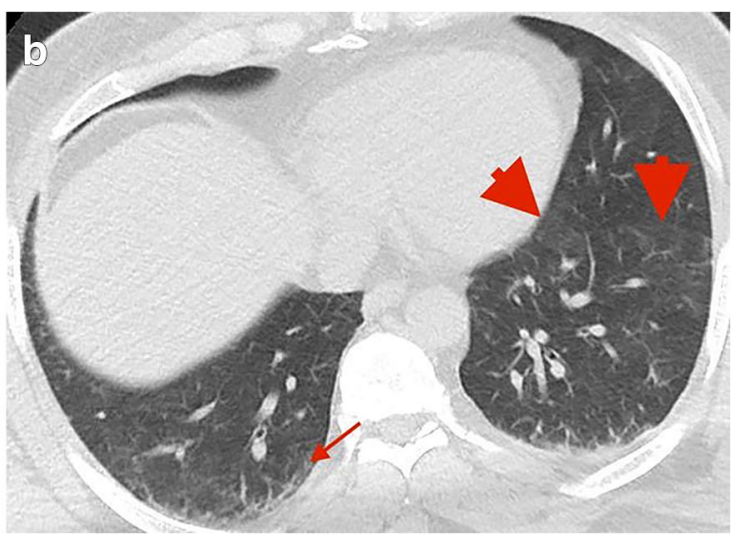

right lower lobe and patchy areas of ground-glass opacities (arrowheads) in the left lower lobe, as residual disease 
Patients with residual lung disease had significantly higher CT severity score when compared to the resolved group. Recent studies on COVID-19 have found CT severity score as a tool for predicting the outcome and mortality on shortterm follow-up [8]. We found that the extent of the lung disease on initial evaluation also potentially can predict the outcome of pulmonary findings in a mid-term follow-up. Thus, patients with more extensive lung involvement may benefit from follow-up CTs and potential management options, given the organizing pneumonia as a consequence of viral infection has the potential to progress to fibrosis [15]. It is worth mentioning that majority of the patients with residual disease had a mild disease in their follow-up CT, based on the CT severity score, even though their initial CT severity score was higher in comparison to patients with the resolved disease. Regarding the pattern of lung involvement, patients with residual disease had GGO or peripheral parenchymal bands or a combination of them in the primary sites of the infection, which may be representative of early fibrotic or interstitial lung disease [18]. Similar findings were observed in some prior studies on longterm follow-up of influenza A pneumonia [24, 25]; however, patients in our study did not show evidences of bronchiectasis, pneumatocele, or bullous cyst formation.

As mentioned, it was revealed that patients with residual disease on follow-up CT experienced higher rate of ICU hospitalization and were in the hospital for a longer duration if admitted. These findings show that critically ill patients with endotracheal intubation throughout their course who spent a longer time in the hospital are more likely to have sequela of disease. It seems that a follow-up examination for this group of patients could be helpful for the identification of chronic lung involvement, which can be done by physical examination, pulmonary function test, or CT imaging. It is worth knowing that Zhao $\mathrm{YM}$ et al. assessed the pulmonary function test in COVID-19 survivors 3 months after discharge, and found that residual abnormalities of pulmonary function were observed in $25.4 \%$ of the patients, mostly demonstrated diffusion reductions in DLCO, which indicated pulmonary fibrosis or a late phase in the course of recovery [26].

More than half of the patients with residual disease on follow-up chest CT had at least one underlying medical condition, significantly higher than the group with the resolved disease. This factor has also been predictive in the acute outcome of the disease in numerous prior studies [27, 28]. It is well-known that some comorbidities like diabetes mellitus, specific pulmonary diseases, heart failure, and malignancy make the patients prone to pneumonia [29]. According to our study, it can be stated that such conditions can also be risk factors or prognostic factors for residual pulmonary sequela of COVID-19 pneumonia.

Researchers have used a few laboratory tests as predictors of mortality in COVID-19 [27, 30]. In the present study, we assessed the laboratory findings for prediction of chronic pulmonary sequela of the disease. Patients with residual disease on follow-up CT had significantly higher WBC count on their initial presentation, and higher occurrence rate of leukocytosis during the course of disease. Also, these patients had overall higher CRP and LDH levels. These laboratory factors should be considered potential predictors of chronic lung sequela, though dedicated statistically more advanced larger studies are warranted.

In our retrospective study, the mean BMI was higher in patients with residual disease although the two groups did not significantly differ in statistical analysis. Multiple prior studies have shown that BMI is a significant risk factor of mortality in patients with COVID-19 pneumonia $[31,32]$ but its role in predicting the mid-term sequela has not been well-assessed on current literature and further studies with larger number of cases are warranted.

There are a few limitations to our study. Our cohort consisted of the patients who had a chest $\mathrm{CT}$ at the time of the admission or presentation and may not reflect the accurate burden of the sequela of the disease in the community. In addition, only 52 patients were enrolled in our study. Larger sample sizes would be more ideal for the study, and allow achieving more details such as the possible effect of therapeutic regimens on the resolution of CT findings. Because the CT protocol in our retrospective study did not include intravenous contrast admission, the development of pulmonary emboli could not be assessed, although in four patients who had subsequent pulmonary $\mathrm{CT}$ angiography, there were no pulmonary emboli. Another limitation of our study was the problem of differentiating dependent atelectasis from fibrosis. When it was patchy and local, we considered it abnormal. However, in some patients, more diffuse subpleural fibrosis may mimic dependent atelectasis, and vice versa. This problem can be definitely resolved by scanning the patient in the both supine and prone position, which warrants a prospective study. Our study was a retrospective analysis from a single institution, and our proposed findings should be confirmed via more statistically advanced studies and larger cohorts. Although all patients received supportive management during their disease course and there was no significant difference between two groups regarding specific medications, there is not still a proven therapeutic drug regimen for COVID-19 pneumonia, which potentially can alter the prognosis and impact of the disease in the lungs. Last but not least, pulmonary function test (PFT) may be used as an additional test or surrogate for CT in mid-term follow-up of COVID-19 pneumonia, although further studies are warranted.

In conclusion, COVID-19 pneumonia patients who have residual disease on their 3-month follow-up CT had more clinically critical admission, spent a longer time in the hospital, demonstrated more extensive parenchymal involvement on their initial $\mathrm{CT}$, had more preexisting medical conditions, and had higher initial WBC and occurrence rate of 
leukocytosis during the course of disease, factors with potential prognostic utility in predicting chronic lung sequela.

\section{Main points}

- Most patients with COVID-19 pneumonia had complete resolution of the disease on a 3-month follow-up.

- Ground-glass opacities, mixed ground-glass and subpleural parenchymal bands, and pure parenchymal bands were the most common $\mathrm{CT}$ findings on a 3-month follow-up, respectively.

- There are some potential predictors for chronic lung disease in COVID-19 pneumonia.

- Extensive lung involvement on initial CT, ICU admission, long duration of hospitalization, underlying medical conditions, and development of leukocytosis during the course of disease are associated with more prevalence of chronic lung sequela of COVID-19.

Acknowledgments We would like to thank Dr. Parastoo Yavari in the Radiology Department of Kashan University of Medical Sciences for her assistance in data collection.

Data availability The data that support the findings of this study are available upon reasonable request.

\section{Compliance with ethical standards}

Conflict of interest The authors declare that they have no conflict of interest.

\section{References}

1. World Health Organization. WHO coronavirus disease (COVID19) dashboard. https://covid19.who.int/ Accessed 3 September 2020

2. Ai T, Yang Z, Hou H, Zhan C, Chen C, Lv W, Tao Q, Sun Z, Xia L (2020) Correlation of chest CT and RT-PCR testing in coronavirus disease 2019 (COVID-19) in China: a report of 1014 cases. Radiology. 26:200642. https://doi.org/10.1148/radiol.2020200642

3. Caruso D, Zerunian M, Polici M, Pucciarelli F, Polidori T, Rucci C, Guido G, Bracci B, de Dominicis C, Laghi A (2020) Chest CT features of COVID-19 in Rome, Italy. Radiology 3:201237201E85. https://doi.org/10.1148/radiol.2020201237

4. Wang W, Xu Y, Gao R, Lu R, Han K, Wu G, Tan W (2020) Detection of SARS-CoV-2 in different types of clinical specimens. Jama. 323(18):1843-1844. https://doi.org/10.1001/jama.2020. 3786

5. Zou L, Ruan F, Huang M, Liang L, Huang H, Hong Z, Yu J, Kang M, Song Y, Xia J, Guo Q (2020) SARS-CoV-2 viral load in upper respiratory specimens of infected patients. N Engl J Med 382(12): 1177-1179. https://doi.org/10.1056/NEJMc2001737

6. Rubin GD, Ryerson CJ, Haramati LB, Sverzellati N, Kanne JP, Raoof S, Schluger NW, Volpi A, Yim JJ, Martin IB, Anderson DJ (2020) The role of chest imaging in patient management during the COVID-19 pandemic: a multinational consensus statement from the Fleischner Society. Chest 158:106-116. https://doi.org/ 10.1016/j.chest.2020.04.003
7. Tabatabaei SMH, Talari H, Moghaddas F, Rajebi H (2020) Computed tomographic features and short-term prognosis of coronavirus disease 2019 (COVID-19) pneumonia: a single-center study from Kashan, Iran. Radiology: Cardiothoracic Imaging 2(2):e200130. https://doi.org/10.1148/ryct.2020200130

8. Francone M, Iafrate F, Masci GM, Coco S, Cilia F, Manganaro L, Panebianco V, Andreoli C, Colaiacomo MC, Zingaropoli MA, Ciardi MR (2020) Chest CT score in COVID-19 patients: correlation with disease severity and short-term prognosis. Eur Radiol 4:16817. https://doi.org/10.1007/s00330-020-07033-y

9. Lu X, Gong W, Peng Z, Zeng F, Liu F (2020) High resolution CT imaging dynamic follow-up study of novel coronavirus pneumonia. Frontiers in Medicine 7:168. https://doi.org/10.3389/fmed.2020. 00168

10. Vernuccio F, Giambelluca D, Cannella R, Lombardo FP, Panzuto F, Midiri M, Cabassa P (2020) Radiographic and chest CT imaging presentation and follow-up of COVID-19 pneumonia: a multicenter experience from an endemic area. Emerg Radiol 11:1-10

11. Tabatabaei SMH, Talari H, Gholamrezanezhad A, Farhood B, Rahimi H, Razzaghi R, Mehri N, Rajebi H (2020) A low-dose chest CT protocol for the diagnosis of COVID-19 pneumonia: a prospective study. Emerg Radiol 13:1-9. https://doi.org/10.1007/s10140020-01838-6

12. Pan Y, Guan H, Zhou S, Wang Y, Li Q, Zhu T, Hu Q, Xia L (2020) Initial CT findings and temporal changes in patients with the novel coronavirus pneumonia (2019-nCoV): a study of 63 patients in Wuhan. China European radiology 13:1-4. https://doi.org/10. 1007/s00330-020-06731-x

13. Li M, Lei P, Zeng B, Li Z, Yu P, Fan B, Wang C, Li Z, Zhou J, Hu S, Liu H (2020) Coronavirus disease (COVID-19): spectrum of CT findings and temporal progression of the disease. Acad Radiol 27: 603-608. https://doi.org/10.1016/j.acra.2020.03.003

14. Pan F, Ye T, Sun P, Gui S, Liang B, Li L, Zheng D, Wang J, Hesketh RL, Yang L, Zheng C (2020) Time course of lung changes on chest CT during recovery from 2019 novel coronavirus (COVID-19) pneumonia. Radiology. 13:200370-200721. https:// doi.org/10.1148/radiol.2020200370

15. Wang Y, Dong C, Hu Y, Li C, Ren Q, Zhang X, Shi H, Zhou M (2020) Temporal changes of CT findings in 90 patients with COVID-19 pneumonia: a longitudinal study. Radiology. 19: 200843-200E64. https://doi.org/10.1148/radiol.2020200843

16. Xiong Y, Sun D, Liu Y, Fan Y, Zhao L, Li X, Zhu W (2020) Clinical and high-resolution CT features of the COVID-19 infection: comparison of the initial and follow-up changes. Investig Radiol 55:332-339. https://doi.org/10.1097/RLI. 0000000000000674

17. Liu N, He G, Yang X, Chen J, Wu J, Ma M, Lu W, Li Q, Cheng T, Huang X (2020 Aug 5) Dynamic changes of chest CT follow-up in coronavirus disease-19 (COVID-19) pneumonia: relationship to clinical typing. BMC Med Imaging 20(1):92. https://doi.org/10. 1186/s12880-020-00491-2

18. Huang Y, Yan Tan C, Wu J, Zhu Chen M, Guo Wang Z, Yun Luo L, Rong Zhou X, Ran Liu X, Ling Huang X, Can Yuan C, Lin Chen C (2020) Impact of coronavirus disease 2019 on pulmonary function in early convalescence phase. Respir Res 21(1):163. https://doi. org/10.1186/s12931-020-01429-6

19. Salehi S, Reddy S, Gholamrezanezhad A (2020) Long-term pulmonary consequences of coronavirus disease 2019 (COVID-19): what we know and what to expect. J Thorac Imaging 35(4):W87-W89. https://doi.org/10.1097/RTI.0000000000000534

20. BMJ Best practice COVID-19 via https://bestpractice.bmj.com/ topics/en-us/3000168/guidelines Accessed Mar 02, 2020

21. Massachusetts General Hospital COVID-19 Treatment Guidance via https://www.massgeneral.org/news/coronavirus/treatmentguidance Version 2.0 4/10/2020 7:00 AM 
22. Hansell DM, Bankier AA, MacMahon H, McLoud TC, Müller NL, Remy J (2008) Fleischner Society: glossary of terms for thoracic imaging. Radiology 246(3):697-722. https://doi.org/10.1148/ radiol.2462070712

23. Simpson S, Kay FU, Abbara S, Bhalla S, Chung JH, Chung M, Henry TS, Kanne JP, Kligerman S, Ko JP, Litt H (2020) Radiological Society of North America Expert Consensus Statement on Reporting Chest CT Findings Related to COVID19. Endorsed by the Society of Thoracic Radiology, the American College of Radiology, and RSNA. Radiology: Cardiothoracic Imaging 2(2): e200152

24. Chen J, Wu J, Hao S, Yang M, Lu X, Chen X, Li L (2017) Long term outcomes in survivors of epidemic influenza A (H7N9) virus infection. Sci Rep 7(1):1-8. https://doi.org/10.1038/s41598-01717497-6

25. Tang XJ, Xi XH, Chen CC, Tang M, Chen SJ, Zhu ZH, Hou DN, $\mathrm{Lu}$ SH, Li T, Bai CX, Song YL (2016) Long-term follow-up of 5 survivors after the first outbreak of human infections with avian Influenza A (H7N9) virus in Shanghai, China. Chinese Medical Journal 129(17):2128. https://doi.org/10.4103/0366-6999.189061

26. Zhao YM, Shang YM, Song WB, Li QQ, Xie H, Xu QF, Jia JL, Li LM, Mao HL, Zhou XM, Luo H, Gao YF, Xu AG (2020) Followup study of the pulmonary function and related physiological characteristics of COVID-19 survivors three months after recovery. EClinicalMedicine. 25:100463

27. Wynants L, Van Calster B, Bonten MM, Collins GS, Debray TP, De Vos M, Haller MC, Heinze G, Moons KG, Riley RD, Schuit E
(2020) Prediction models for diagnosis and prognosis of COVID19 infection: systematic review and critical appraisal. Bmj. 369. https://doi.org/10.1136/bmj.m1328

28. Guan WJ, Liang WH, Zhao Y, Liang HR, Chen ZS, Li YM, Liu XQ, Chen RC, Tang CL, Wang T, Ou CQ (2020) Comorbidity and its impact on 1590 patients with COVID-19 in China: a nationwide analysis. Eur Respir J 1;55(5). doi: https://doi.org/10.1183/ 13993003.00547-2020

29. Franquet $\mathrm{T}$ (2011) Imaging of pulmonary viral pneumonia. Radiology. 260(1):18-39. https://doi.org/10.1148/radiol.11092149

30. Shang Y, Liu T, Wei Y, Li J, Shao L, Liu M, Zhang Y, Zhao Z, Xu H, Peng Z, Wang X, Zhou F (2020) Scoring systems for predicting mortality for severe patients with COVID-19, EClinicalMedicine Available at SSRN 3582752, https://doi.org/10.1016/j.eclinm. 2020.100426

31. Hussain A, Mahawar K, Xia Z, Yang W, Shamsi EH (2020) Obesity and mortality of COVID-19. Meta-analysis. Obesity research \& clinical practice. doi: https://doi.org/10.1016/j.orcp. 2020.07.002

32. Pettit NN, MacKenzie EL, Ridgway J, Pursell K, Ash D, Patel B, Pho MT (2020) Obesity is associated with increased risk for mortality among hospitalized patients with COVID-19. Obesity. 28: 1806-1810. https://doi.org/10.1002/oby.22941

Publisher's note Springer Nature remains neutral with regard to jurisdictional claims in published maps and institutional affiliations. 